Subgroup Consistent Poverty Indices

\author{
James E. Foster; Anthony F. Shorrocks
}

Econometrica, Vol. 59, No. 3. (May, 1991), pp. 687-709.

Stable URL:

http://links.jstor.org/sici?sici=0012-9682\%28199105\%2959\%3A3\%3C687\%3ASCPI\%3E2.0.CO\%3B2-Y

Econometrica is currently published by The Econometric Society.

Your use of the JSTOR archive indicates your acceptance of JSTOR's Terms and Conditions of Use, available at

http://www.jstor.org/about/terms.html. JSTOR's Terms and Conditions of Use provides, in part, that unless you have obtained prior permission, you may not download an entire issue of a journal or multiple copies of articles, and you may use content in the JSTOR archive only for your personal, non-commercial use.

Please contact the publisher regarding any further use of this work. Publisher contact information may be obtained at http://www.jstor.org/journals/econosoc.html.

Each copy of any part of a JSTOR transmission must contain the same copyright notice that appears on the screen or printed page of such transmission.

The JSTOR Archive is a trusted digital repository providing for long-term preservation and access to leading academic journals and scholarly literature from around the world. The Archive is supported by libraries, scholarly societies, publishers, and foundations. It is an initiative of JSTOR, a not-for-profit organization with a mission to help the scholarly community take advantage of advances in technology. For more information regarding JSTOR, please contact support@jstor.org. 


\title{
SUBGROUP CONSISTENT POVERTY INDICES
}

\author{
By James E. Foster And Anthony F. Shorrocks ${ }^{1}$
}

\begin{abstract}
It seems desirable that the overall level of poverty should fall whenever poverty decreases within some subgroup of the population and is unchanged outside that group. Yet this simple and attractive property, which we call "subgroup consistency," is violated by many of the poverty indices suggested in recent years. This paper characterizes the class of subgroup consistent poverty indices, and identifies the special features associated with this property.
\end{abstract}

KEYwORDs: Poverty measurement, decomposability, separability.

\section{INTRODUCTION AND SUMMARY}

ONE OF THE MOST APPEALING PROPERTIES of poverty indices suggested in recent years is a simple consistency axiom which requires the overall level of poverty to fall if a subgroup of the population experiences a reduction in poverty, while poverty in the rest of the population remains unchanged. ${ }^{2}$ This property-which we term "subgroup consistency"-is desirable for a number of reasons. From a practical point of view, it is needed to coordinate the effects of a decentralized strategy towards poverty alleviation. For a decentralized strategy typically involves a collection of activities targeted at specific subgroups or regions of the country. If the poverty indicator is not subgroup consistent, we may be faced with a situation in which each local effort achieves its objective of reducing poverty within its targeted group, and yet the level of poverty in the population as a whole increases. Subgroup consistency may therefore be viewed as an essential counterpart to a coherent poverty program.

Subgroup consistency may also be regarded as a natural analogue of the monotonicity condition of Sen (1976), since monotonicity requires that aggregate poverty fall (or, at least, does not increase) if one person's poverty is reduced, ceteris paribus, while subgroup consistency demands that aggregate poverty fall if one subgroup's poverty is reduced, ceteris paribus. Furthermore, subgroup consistency is closely related to the property of "decomposability," which allows aggregate poverty to be expressed as a population-share weighted average of subgroup poverty levels, and hence facilitates the disaggregated analysis of poverty by region or ethnic group of the type undertaken by Anand (1983). As it happens, the traditional poverty indices used by Anand and others - the headcount ratio (the fraction of the population that is poor) and the

\footnotetext{
${ }^{1}$ Earlier versions of this paper were presented at a conference on Measurement and Modelling in Economics at Nuffield College, Oxford, in May, 1987, and at the Econometric Society Meetings in Bologna in 1988. We have greatly benefited from suggestions from Chuck Blackorby, Terence Gorman, Aldi Hagenaars, King-Tim Mak, Bill Novshek, Tom Stoker, and two anonymous referees.

${ }^{2}$ The notion of subgroup consistency was first discussed by Foster, Greer, and Thorbecke (1984). Their "subgroup monotonicity" axiom is slightly weaker than subgroup consistency, since it requires the distribution in the rest of the population (rather than just its poverty value) to remain the same. Shorrocks (1988) analyzes a similar property in the context of inequality measures.
} 
aggregate poverty gap (the amount needed to raise all poor to the poverty line) -are both subgroup consistent and decomposable. But inspection of the many poverty measures that have been proposed in the years since Sen (1976) first pointed the way to proceed, reveals that few of the recent "distribution-sensitive" poverty indices satisfy either of these properties.

The Sen index is a case in point. ${ }^{3}$ Consider a country consisting of two regions with identical income distributions $(1,9,9,9,20)$. When the poverty line is set at 10 , the two regions and the aggregate population share a poverty level of 0.36 according to the Sen index. However, if the income distribution in region 1 changes to $(4,5,9,10,20)$, then région 1 poverty falls to 0.35 while poverty in the overall population rises to 0.365 . By the subgroup consistency criterion, therefore, the Sen index is found wanting. An even more striking illustration of inconsistency is provided by some of the indices suggested by Blackorby and Donaldson (1980). ${ }^{4}$ For they allow a policy which completely eliminates poverty in one region, without disturbing the incomes outside that region, to result in a rise in the level recorded for overall poverty. In contrast, no example of inconsistency can be found for the families of indices proposed by Chakravarty (1983a) or Foster, Greer, and Thorbecke (1984).

In this paper we characterize the class of subgroup consistent poverty indices and, in doing so, identify the particular features associated with this property. Our first series of results, given in Section 4, concern the case in which the poverty line has a fixed and constant value. Using a strong continuity assumption, we find that every subgroup consistent poverty index can be transformed into a "canonical" index with a special additive form. In this representation, poverty can be interpreted as the average level of deprivation in society, where an individual's deprivation is zero if income is above the poverty line, and rises continuously (or at least does not decrease) as income falls. Furthermore, each of these "canonical" indices is decomposable. Hence, we conclude that every continuous subgroup consistent poverty index is an increasing transformation of a continuous decomposable index. We go on to show that, by relaxing the continuity assumption, we gain only transformations of the headcount ratio, or of some combination of the headcount ratio and a canonical index.

In Section 5 we allow the poverty line to change, and focus on two classes of indices defined by certain invariance properties. First, we characterize the subset of continuous subgroup consistent indices which are "relative" in the sense that poverty is invariant to a proportionate increase in the poverty line and all incomes. We then repeat the exercise for the subset of "absolute" indices, which have the property that the addition of the same absolute amount to the poverty line and all incomes leaves the index value unchanged. These invariance criteria place special constraints on the form of the individual deprivation function in the decomposable representation. In the case of relative

\footnotetext{
${ }^{3}$ The Sen index is defined by $S=H\left[I+(1-I) G_{p}\right]$, where $H$ is the headcount ratio, $I$ is the income gap ratio (the average shortfall of the poor as a proportion of the poverty line income), and $G_{p}$ is the Gini coefficient among the poor; see Sen (1976).

${ }^{4}$ One of these indices is defined below in (11).
} 
indices, individual deprivation is a function of income expressed as a multiple of the poverty line value, while for absolute indices, individual deprivation is a function of the income shortfall. We examine whether these two subsets of subgroup consistent indices have any members in common, and discover that only monotonic transformations of the headcount ratio satisfy both requirements.

Our final results revolve around a concept that is a natural generalization of the requirement that an index be both relative and absolute. We say that a relative index and an absolute index are "compatible" if, at any fixed poverty line, they give the same ranking of distributions, although not necessarily the same values. We then ask if there exist compatible pairs of indices that are subgroup consistent (in addition to the headcount ratio, which is clearly compatible with itself). It is shown that if a pair of continuous subgroup consistent indices is compatible, then the relative index must be an increasing transformation of a member of the Foster et al. class.

\section{PRELIMINARY NOTATION AND DEFINITIONS}

We consider discrete income distributions represented by vectors drawn from the set $\mathscr{D}:=\cup_{n=1}^{\infty} D^{n}$, where $D:=\langle a, b\rangle$ is some nondegenerate real interval, and $D^{n}$ is the set of all $n$-tuples of elements from $D$. It may be helpful to regard $D$ as the positive reals $\mathbb{R}_{++}$, but in general we do not require incomes to be positive or to have an unbounded range. For any given poverty line $z \in D$ and distribution $x \in \mathscr{D}$, we define as poor all incomes $x_{i} \leq z$, so the "poverty domain" is $D_{p}(z):=\{t \in D \mid t \leq z\}$. $^{5}$ The population size corresponding to $x$ is denoted by $n(x)$, the number of poor incomes by $q(x ; z)$, and the average income of the poor by $\mu_{p}(x ; z)$. We say that $x \in \mathscr{D}$ is obtained from $y \in \mathscr{D}$ by a permutation if $x=y \Pi$ for some permutation matrix $\Pi$, and let the ordered version $\hat{x}$ denote the permutation of $x$ for which $\hat{x}_{1} \leq \hat{x}_{2} \leq \cdots \leq \hat{x}_{n(x)}$. Finally we say that $x \in \mathscr{D}$ is obtained from $y \in \mathscr{D}$ by a $(k$-)replication if $n(x)=k \cdot n(y)$ and $x=(y, y, \ldots, y)$ for some positive integer $k$; and by an increment to a poor (resp. nonpoor) person if $x_{i}=y_{i}$ for all $i \neq j$ and $x_{j}>y_{j}$ for some $y_{j} \leq z$ (resp. $\left.y_{j}>z\right)$.

A poverty index is a function $P: \mathscr{D} \times D \rightarrow \mathbb{R}$ whose typical value $P(x ; z)$ indicates the poverty level associated with the distribution $x$ and the poverty line $z$. Examples include the headcount ratio $H(x ; z):=q(x ; z) / n(x)$, the income gap ratio $I(x ; z):=1-\mu_{p}(x ; z) / z$, the per-capita income gap indices $G(x ; z):=H(x ; z) I(x ; z)$ and $A(x ; z):=z G(x ; z)$, the Sen (1976) index, and the classes of indices proposed by Blackorby and Donaldson (1980), Clark, Hemming, and Ulph (1981), Chakravarty (1983a, 1983b), and Foster et al.

\footnotetext{
${ }^{5}$ Many results in this paper would be unaffected if we adopted the alternative definition of the poor, $x_{\imath}<z$. For example, all the results involving continuous poverty indices carry over immediately.
} 
(1984). We assume throughout that the index $P$ satisfies the following five basic properties for any given poverty line $z \in D$ :

(1) Symmetry: $P(x ; z)=P(y ; z)$ whenever $x \in \mathscr{D}$ is obtained from $y \in \mathscr{D}$ by a permutation.

(2) Replication Invariance: $P(x ; z)=P(y ; z)$ whenever $x \in \mathscr{D}$ is obtained from $y \in \mathscr{D}$ by a replication.

(3) Monotonicity: $P(x ; z) \leq P(y ; z)$ whenever $x \in \mathscr{D}$ is obtained from $y \in \mathscr{D}$ by an increment to a poor person.

(4) Focus: $P(x ; z)=P(y ; z)$ whenever $x \in \mathscr{D}$ is obtained from $y \in \mathscr{D}$ by an increment to a nonpoor person.

(5) Restricted Continuity: $P(x ; z)$ is continuous as a function of $x_{i}$ on $D_{p}(z)$.

These axioms are now quite standard and are satisfied by all the poverty indices mentioned above. ${ }^{6}$ Symmetry allows incomes to be re-ordered without affecting the poverty value. Replication invariance ensures that the index views poverty in per-capita terms, so that comparisons across different-sized populations are meaningful. Monotonicity requires that the index does not register an increase in poverty when one or more poor incomes are raised, while the focus axiom implies that the index is independent of the income levels of the nonpoor. Finally, restricted continuity says that the index is a continuous function of poor incomes. $^{7}$

These five properties are the only restrictions implicit in our use of the term "poverty index." We will, however, refer to a number of other properties during the course of our analysis. For instance, we will say that a poverty index is trivial if its value is the same for all distributions and all poverty lines.

Definition: A poverty index $P$ is trivial if $P(x ; z)=P\left(x^{\prime} ; z^{\prime}\right)$ for all $x, x^{\prime} \in \mathscr{D}$ and all $z, z^{\prime} \in D$.

We will also find it helpful to specify a stronger version of (5) which requires $P$ to be continuous on the entire income domain $\mathscr{D}$.

Definition: A poverty index $P$ is continuous if, for every $z \in D, P(x ; z)$ is continuous as a function of $x$ on $\mathscr{D}$.

This continuity property is a good deal less general than its restricted counterpart, since it removes from consideration all those indices which experience an abrupt change when the number of poor persons changes: for example, $H, I$, and the Sen index. For this reason, only restricted continuity (5) is maintained throughout the paper.

\footnotetext{
${ }^{6}$ Other indices not mentioned in the text include those of Kakwani (1980) and Thon (1979), which violate replication invariance, and that of Takayama (1979), which violates monotonicity.

${ }^{7}$ For discussions of the properties of poverty indices, see Foster (1984) and Donaldson and Weymark (1986).
} 


\section{SUBGROUP CONSISTENCY}

The objective of this paper is to investigate a property which ensures that the aggregate poverty value does not respond perversely to changes in the level of poverty within subgroups of the population. More precisely, we imagine a situation in which the population is partitioned into two fixed-sized subgroups, and that poverty increases in one and stays constant in the other. Our subgroup consistency axiom then requires the overall poverty level to increase.

Definition: A poverty index $P$ is subgroup consistent if, for every $z \in D$ and any $x, x^{\prime}, y, y^{\prime} \in \mathscr{D}$ for which $n(x)=n\left(x^{\prime}\right)$ and $n(y)=n\left(y^{\prime}\right)$, we have

$$
P(x, y ; z)>P\left(x^{\prime}, y^{\prime} ; z\right)
$$

whenever

$$
P(x ; z)>P\left(x^{\prime} ; z\right) \quad \text { and } \quad P(y ; z)=P\left(y^{\prime} ; z\right) .
$$

Before considering the implications of this axiom, let us take note of the conditions under which it applies. A principal restriction is the one placed on subgroup population sizes: there is no population growth or migration between the two subgroups. In the absence of this constraint the property would be too far reaching, as can be seen from a simple example. Suppose that poverty is initially higher in region 2 than in region 1 . If a representative sample of families shifts from region 2 to region 1, poverty might well go up in 1 and stay the same in 2 . Yet requiring overall poverty to increase in this instance would be absurd since the aggregate income distribution is unchanged. Subgroup sizes are fixed in the axiom to rule out any changes in subgroup poverty due entirely to population shifts.

The axiom also places constraints on the number of subgroups and the precise way their poverty levels alter. However, these conditions are less restrictive than may appear at first glance. Although any single use of the axiom requires a partition of the population into exactly two subgroups in which poverty levels are respectively increased and unchanged, repeated application allows it to influence a much broader set of circumstances. For instance, the requirement that poverty be unchanged in the second subgroup can be replaced by the weak inequality $P(y ; z) \geq P\left(y^{\prime} ; z\right)$ without affecting the axiom. And the number of (fixed-sized) subgroups may also be extended to any number of subgroups $K \geq 2$ by requiring that aggregate poverty increase if poverty does not fall in any subgroup and increases in at least one. For simplicity, however, we have chosen to state the axiom for the two subgroup case.

The subgroup consistency property is closely allied to the stronger condition of decomposability defined by Foster et al. (1984) as follows:

Definition: A poverty index $P$ is decomposable if, for every $K \geq 2$ and any $x^{k} \in \mathscr{D}, k=1, \ldots, K$,

$$
P\left(x^{1}, \ldots, x^{K} ; z\right)=\sum_{k=1}^{K} \omega_{k} P\left(x^{k} ; z\right),
$$

where $\omega_{k}=n\left(x^{k}\right) / n(x)$. 
For decomposable indices, overall poverty is a weighted average of subgroup poverty, where the weights are given by the subgroup population shares. If these population shares stay constant, an increase in the level of poverty in one subgroup will increase overall poverty. So a decomposable index must be subgroup consistent. It follows immediately that the set of subgroup consistent indices includes the headcount ratio $H$, the per-capita income gaps $G$ and $A$, and the families of decomposable indices proposed by Chakravarty (1983a),

$$
Q_{\beta}(x ; z):=\frac{1}{n(x)} \sum_{i=1}^{q(x ; z)}\left[1-\left(\frac{\hat{x}_{i}}{z}\right)^{\beta}\right], \quad 0<\beta<1,
$$

and by Foster et al. (1984),

$$
P_{\alpha}(x ; z):=\frac{1}{n(x)} \sum_{i=1}^{q(x ; z)}\left[1-\frac{\hat{x}_{i}}{z}\right]^{\alpha}, 0 \leq \alpha .
$$

The list of poverty measures mentioned earlier also contains cases of indices that are subgroup consistent but not decomposable. The second family suggested by Clark et al. provides an example. This family is defined as

$$
C_{\beta}(x ; z)=1-\left[f_{\beta}(x ; z)\right]^{1 / \beta}, \quad \beta<1,
$$

where

$$
f_{\beta}(x ; z):=\frac{1}{n(x)} \sum_{i=1}^{n(x)} \min \left(\frac{x_{i}}{z}, 1\right)^{\beta} .
$$

To confirm that $C_{\beta}$ is subgroup consistent, observe that

$$
C_{\beta}(x, y ; z)=1-\left[\frac{n(x)}{n(x, y)} f_{\beta}(x ; z)+\frac{n(y)}{n(x, y)} f_{\beta}(y ; z)\right]^{1 / \beta} .
$$

So if $C_{\beta}(x ; z)$ rises and $C_{\beta}(y ; z)$ remains the same, then $f_{\beta}(x ; z)$ falls and $f_{\beta}(y ; z)$ remains the same, which means that $C_{\beta}(x, y ; z)$ rises.

Almost all the other indices on our list are not subgroup consistent, ${ }^{8}$ and at least two specific sources of failure can be identified. The Sen index violates the axiom because of its connection with the Gini measure of inequality and its reliance on rank-order weighting. This can be seen from the example given in the introduction, where the change in the first region's distribution leaves the number of poor and their average income intact, but alters the inequality among the poor. Subgroup consistency is violated because the change in region 1's income distribution is a "composite transfer," involving a progressive transfer among the poorest poor and a regressive transfer among the richer poor. After the change, the Gini coefficient indicates a lower level of inequality among the poor in region 1; hence poverty falls in that region according to the Sen index.

\footnotetext{
${ }^{8}$ Violators include $I, S$, members of the classes of Blackorby and Donaldson (1980) and Chakravarty (1983b), and the first family of Clark et al. (1981).
} 
However, all the poor incomes in region 2 are located at the upper end of the poor range. Since the impact of a transfer on the Gini coefficient depends upon the number of incomes between the donor and recipient, this causes the regressive transfer to have a larger effect when the regions are combined. Hence, the aggregate Gini among the poor is higher as a result of the composite transfer, and so is aggregate poverty according to the Sen index.

Other indices violate subgroup consistency because of their averaging or normalization factors. This is the case, for instance, with the income gap ratio $I(x ; z)=\left(z-\mu_{p}\right) / z=(1 / q) \sum_{i=1}^{q}\left(z-\hat{x}_{i}\right) / z$. Due to its use of $1 / q$ as a normalization factor, the income gap ratio is concerned only with the average income of the poor $\mu_{p}$, not with their number $(q)$, or the total population size $(n)$. And since the average income of the poor can rise in a subgroup and fall overall, subgroup consistency can easily be violated. For example, if $z=10, x=(1,20)$, $x^{\prime}=(2,2)$, and $y=(10,10)$, we find that $I(x ; z)=0.9$ and $I\left(x^{\prime} ; z\right)=0.8$. But $I(x, y ; z)=0.3<I\left(x^{\prime}, y ; z\right)=0.4$, reversing the required order. This inconsistency would not arise if the gaps were normalized by the factor $1 / n$ rather than $1 / q$, thus obtaining the subgroup consistent index $G=H I$.

The Blackorby and Donaldson (1980) indices can exhibit even more striking inconsistencies for the same reason. For instance, when the parameter takes the value $\varepsilon=-1$, their index becomes

$$
B_{\varepsilon}(x ; z)=H\left(z-\mu_{p}^{h}\right) / z
$$

where

$$
\mu_{p}^{h}:=\left[\frac{1}{q(x ; z)} \sum_{i=1}^{q(x ; z)}\left(1 / \hat{x}_{i}\right)\right]^{-1} .
$$

Using $x$ and $y$ defined above we obtain $B_{\varepsilon}(x ; z)=0.45$ and $B_{\varepsilon}(y ; z)=0$. So we might reasonably expect that $B_{\varepsilon}(x, x ; z)>B_{\varepsilon}(x, y ; z)$. However, $B_{\varepsilon}(x, x ; z)=$ 0.45 and $B_{\varepsilon}(x, y ; z)=0.56$. Thus, eliminating poverty in the second subgroup results in an overall increase in poverty! Once again, simply changing the normalization factors leads to a subgroup consistent index. ${ }^{9}$

\section{THE CLASS OF SUBGROUP CONSISTENT POVERTY INDICES}

In this section we obtain a characterization of subgroup consistent indices. To simplify the notation we regard the poverty line $z$ as fixed and omit it as an argument of the poverty index and the other functions and sets defined earlier. We begin by observing that a subgroup consistent poverty index induces an ordering on each $D^{n}$ that is strictly separable (in the sense of Gorman (1968)) in each partition of incomes. By symmetry, this amounts to showing that for any positive integers $k$ and $l$ satisfying $k+l=n$, and all $x, x^{\prime} \in D^{k}$ and $y, y^{\prime} \in D^{l}$,

\footnotetext{
${ }^{9}$ This particularly bad example of inconsistency would not occur if the $H$ were removed from the definition of $B_{\varepsilon}$, and the factor $1 / n$ replaced $1 / q$ in the expression for $\mu_{p}^{h}$.
} 
we have

$$
P(x, y) \geq P\left(x^{\prime}, y\right) \text { implies } P\left(x, y^{\prime}\right) \geq P\left(x^{\prime}, y^{\prime}\right) .
$$

This will now be verified.

Suppose that $P(x, y) \geq P\left(x^{\prime}, y\right)$. Then by subgroup consistency we have $P(x) \geq P\left(x^{\prime}\right)$. If $P(x)>P\left(x^{\prime}\right)$, we immediately obtain $P\left(x, y^{\prime}\right)>P\left(x^{\prime}, y^{\prime}\right)$ by subgroup consistency. Alternatively, if $P(x)=P\left(x^{\prime}\right)$ then we cannot have $P\left(x, y^{\prime}\right)<P\left(x^{\prime}, y^{\prime}\right)$, since this would imply $P\left(x, y^{\prime}, x^{\prime}\right)<P\left(x^{\prime}, y^{\prime}, x\right)$ by subgroup consistency, contradicting symmetry. Thus in either case we obtain $P\left(x, y^{\prime}\right) \geq$ $P\left(x^{\prime}, y^{\prime}\right)$, so $(12)$ is true.

Our first objective is to derive the functional form of continuous subgroup consistent indices. Applying standard results on separability (Gorman (1968), Blackorby et al. (1978)) to (12), we find that for every integer $n \geq 3$

$$
P(x)=\tilde{F}_{n}\left[\sum_{i=1}^{n} \tilde{\phi}_{n}\left(x_{i}\right)\right] \quad \text { for all } x \in D^{n},
$$

where $\tilde{\phi}_{n}$ is continuous and $\tilde{F}_{n}$ is continuous and increasing. ${ }^{10}$ Setting $\phi_{n}(t):=$ $n\left[\tilde{\phi}_{n}(t)-\tilde{\phi}_{n}(z)\right]$ for $t \in D$ and $F_{n}(u):=\tilde{F}_{n}\left[u+n \tilde{\phi}_{n}(z)\right]$ for all $u \in \phi_{n}(D)$, we obtain

$$
P(x)=F_{n}\left[\frac{1}{n} \sum_{i=1}^{n} \phi_{n}\left(x_{i}\right)\right] \quad \text { for each } n \geq 3 \text { and every } x \in D^{n},
$$

where $\phi_{n}: D \rightarrow \mathbb{R}$ is continuous; $\phi_{n}(z)=0$; and $F_{n}: \phi_{n}(D) \rightarrow \mathbb{R}$ is continuous and increasing.

The replication invariance condition (2) allows us to choose the functions $\phi_{n}$ and $F_{n}$ in (14) to be independent of $n$, and to extend (14) to the cases $n=1$ and $n=2$. Let $\phi:=\phi_{4}, F:=F_{4}$, and consider any $m:=4 n$, where $n$ is a positive integer. For any $t \in D$, define $w:=(t, t, t, t)$ and its replication $w^{\prime} \in D^{m}$. From (2) and (14) we deduce

$$
F[\phi(t)]=P(w)=P\left(w^{\prime}\right)=F_{m}\left[\phi_{m}(t)\right] \text { for every } t \in D .
$$

Substituting (15) into (14) then yields

$$
\begin{aligned}
F^{-1}[P(x)] & =F^{-1}\left[F_{m}\left[\frac{1}{m} \sum_{i=1}^{m} F_{m}^{-1}\left[F\left[\phi\left(x_{i}\right)\right]\right]\right]\right] \\
& =G_{m}^{-1}\left[\frac{1}{m} \sum_{i=1}^{m} G_{m}\left[\phi\left(x_{i}\right)\right]\right] \quad \text { for every } x \in D^{m},
\end{aligned}
$$

where $G_{m}(u):=F_{m}^{-1}[F(u)]$ is continuous and increasing on $\phi(D)$; and $G_{4}(u)=u$.

${ }^{10}$ Gorman's requirement that each sector be essential is clearly satisfied if $P$ is not constant on $D^{n}$. In the case where $P$ is constant, we can express $P$ in this form without having to appeal to Gorman's result. 
Now consider any $y \in D^{2}$ and its replications $y^{\prime} \in D^{4}$ and $y^{\prime \prime} \in D^{m}$. Setting $u_{i}=\phi\left(y_{i}\right)$, and using (2) and (16), we obtain,

$$
\begin{aligned}
F^{-1}\left[P\left(y^{\prime \prime}\right)\right] & =G_{m}^{-1}\left[\frac{1}{m} \sum_{i=1}^{m} G_{m}\left[\phi\left(y_{i}^{\prime \prime}\right)\right]\right]=G_{m}^{-1}\left[\frac{1}{2} G_{m}\left(u_{1}\right)+\frac{1}{2} G_{m}\left(u_{2}\right)\right], \\
& =F^{-1}\left[P\left(y^{\prime}\right)\right]=G_{4}^{-1}\left[\frac{1}{2} G_{4}\left(u_{1}\right)+\frac{1}{2} G_{4}\left(u_{2}\right)\right]=\frac{1}{2}\left(u_{1}+u_{2}\right) .
\end{aligned}
$$

Hence

$$
\frac{1}{2} G_{m}\left(u_{1}\right)+\frac{1}{2} G_{m}\left(u_{2}\right)=G_{m}\left[\frac{1}{2}\left(u_{1}+u_{2}\right)\right] \text { for all } u_{1}, u_{2} \in \phi(D) .
$$

The solution to this Jensen equation (Aczel $\left(1966\right.$, p. 46)) implies $G_{m}(u)=r_{m} u$ $+s_{m}$ for some constants $r_{m}$ and $s_{m},{ }^{11}$ which upon substitution into (16) gives

$$
F^{-1}[P(x)]=\frac{1}{m} \sum_{i=1}^{m} \phi\left(x_{i}\right) \quad \text { for every } x \in D^{m},
$$

whenever $m=4 n$ and $n$ is a positive integer.

Finally, for each $n \geq 1$ consider any $x \in D^{n}$ and its replication $x^{\prime} \in D^{4 n}$. From (2) and (19) we obtain

$$
F^{-1}[P(x)]=F^{-1}\left[P\left(x^{\prime}\right)\right]=\frac{1}{4 n} \sum_{i=1}^{4 n} \phi\left(x_{i}^{\prime}\right)=\frac{1}{n} \sum_{i=1}^{n} \phi\left(x_{i}\right) .
$$

Therefore

$$
P(x)=F\left[\frac{1}{n} \sum_{i=1}^{n} \phi\left(x_{i}\right)\right] \text { for each } n \geq 1 \text { and every } x \in D^{n},
$$

where $\phi: D \rightarrow \mathbb{R}$ is continuous; $\phi(z)=0$; and $F: \phi(D) \rightarrow \mathbb{R}$ is continuous and increasing. In addition, the monotonicity and focus axioms (3) and (4) imply that $\phi$ is nonincreasing on $D$; and $\phi(t)=0$ for $t \geq z$.

These conditions on $F$ and $\phi$ along with expression (21) form a set of necessary conditions for $P$ to be a continuous and subgroup consistent poverty index. It is easily demonstrated that they are also sufficient. For (21) and the restrictions on $F$ and $\phi$ ensure that $\mathrm{P}$ satisfies symmetry, replication invariance, monotonicity, continuity, and the focus axiom. Furthermore, we see immediately that

$$
P^{\phi}(x):=\frac{1}{n(x)} \sum_{i=1}^{n(x)} \phi\left(x_{i}\right) \quad \text { for all } x \in \mathscr{D}
$$

is decomposable, and hence $P=F\left[P^{\phi}\right]$ is subgroup consistent. We may therefore state the first of our main results.

\footnotetext{
${ }^{11}$ Technically speaking, Aczel's solution only applies when $\phi(D)$ is a nondegenerate interval. However, if $\phi(D)$ is degenerate, the solution to the functional equation obviously has the postulated form. We will use this observation again several times without explicit reference.
} 
Proposition 1: $P: \mathscr{D} \rightarrow \mathbb{R}$ is a continuous, subgroup consistent poverty index if and only if there exist $\phi: D \rightarrow \mathbb{R}$ and $F: \phi(D) \rightarrow \mathbb{R}$ such that

$$
P(x)=F\left[\frac{1}{n(x)} \sum_{i=1}^{n(x)} \phi\left(x_{i}\right)\right] \text { for all } x \in \mathscr{D},
$$

where $F$ is continuous and increasing; $\phi$ is continuous and nonincreasing; and $\phi(t)=0$ for all $t \geq z$.

The poverty indices $P^{\phi}$ given in (22) will play a central role in our subsequent analysis. When $\phi$ satisfies the conditions listed in (23), we will say that the corresponding expression $P^{\phi}$ is a canonical index. So Proposition 1 tells us that any continuous subgroup consistent poverty index must be a continuous and increasing transformation of a canonical index. Furthermore, it may be seen that canonical indices essentially form the subclass of continuous decomposable poverty indices. For if $P$ is continuous and decomposable, then $P$ is also subgroup consistent, and applying Proposition 1 and the definition of decomposability to $x \in D^{2}$, we obtain

$$
\begin{aligned}
F\left[\frac{1}{2} \phi\left(x_{1}\right)+\frac{1}{2} \phi\left(x_{2}\right)\right] & =P(x)=\frac{1}{2} P\left(x_{1}\right)+\frac{1}{2} P\left(x_{2}\right) \\
& =\frac{1}{2} F\left[\phi\left(x_{1}\right)\right]+\frac{1}{2} F\left[\phi\left(x_{2}\right)\right] .
\end{aligned}
$$

Setting $u_{i}=\phi\left(x_{i}\right)$ then yields the Jensen equation

$$
\frac{1}{2} F\left(u_{1}\right)+\frac{1}{2} F\left(u_{2}\right)=F\left[\frac{1}{2}\left(u_{1}+u_{2}\right)\right] \text { for all } u_{1}, u_{2} \in \phi(D),
$$

where $F$ is increasing. This implies $F(u)=r u+c$ for some constant $c$ and some positive constant $r$, which on substitution into (23) yields the following corollary.

Corollary 1: $P$ is a continuous decomposable poverty index if and only if $P=P^{\phi}+c$ for some canonical index $P^{\phi}$ and some constant $c$.

This result indicates the wide range of decomposable poverty indices. The two parametric families provided in the literature by Chakravarty (1983a) and Foster et al. (1984) correspond to $\phi(t)=1-(t / z)^{\beta}$ and $\phi(t)=[1-(t / z)]^{\alpha}$ for $t \leq z$. But these are just two examples of the vast array of possible functional forms for $\phi$.

Combining Proposition 1 with Corollary 1, we immediately obtain a second corollary.

Corollary 2: $P$ is a continuous, subgroup consistent poverty index if and only if $P$ is a continuous, increasing transformation of a continuous, decomposable poverty index.

Subgroup consistency thus provides a means of justifying the use of decomposable poverty measures. For, corresponding to each continuous subgroup 
consistent index there is a continuous decomposable index which ranks distributions in precisely the same way. ${ }^{12}$

One of the attractions of the canonical form $P^{\phi}$ is the interesting interpretation of poverty which it offers. The function $\phi$ may be regarded as a measure of deprivation-a nonincreasing function which attains its minimum level of 0 at $z$, and maintains this value at all nonpoor incomes. The index $P^{\phi}$ then takes overall poverty to be the average deprivation in society as a whole. $P^{\phi}$ may also be expressed in terms of the average deprivation among the poor. For, since $\phi(t)=0$ for $t>z$, we may write

$$
P^{\phi}(x)=H(x) \Phi(x) \text { for all } x \in \mathscr{D},
$$

where $H$ is the headcount ratio and $\Phi: \mathscr{D} \rightarrow \mathbb{R}$ is an average deprivation function among the poor defined by

$$
\Phi(x)= \begin{cases}\frac{1}{q(x)} \sum_{i=1}^{q(x)} \phi\left(\hat{x}_{i}\right) & \text { if } q(x)>0, \\ 0 & \text { if } q(x)=0 .\end{cases}
$$

For instance, if we take the income gap ratio $I(x)$ as the average deprivation among the poor, we obtain the useful decomposable index $G=H I$ mentioned by Sen (1976) and others. If instead of using the normalized shortfall $\left(z-x_{i}\right) / z$ as the individual deprivation function, we take it to some power $\alpha>0$, then the resulting average deprivation function $I_{\alpha}$ may be regarded as a generalized income gap ratio which is more sensitive to the poorest among the poor as $\alpha$ grows larger. And the associated decomposable poverty indices $P_{\alpha}$ are members of the class of Foster et al. (1984).

Proposition 1 and its corollaries depend crucially on the assumption of continuity. We now investigate how these results change when this assumption is dropped. One immediate consequence is that increasing transformations of the headcount ratio join the continuous increasing transformations of $P^{\phi}$ as admissible indices. And since any sum of decomposable indices is decomposable, the class expands further to include continuous increasing transformations of convex combinations of $H$ and $P^{\phi}$. In fact, we will show that any subgroup consistent index $P$ may be expressed as a function of $H$ and some $P^{\phi}$.

We begin by defining $\mathscr{D}_{p}:=\left\{x \in \mathscr{D} \mid x_{i} \leq z \forall i\right\}$ and $\mathscr{D}_{r}:=\left\{x \in \mathscr{D} \mid x_{i}>z \forall i\right\}$ to be the sets of poverty income distributions and nonpoor income distributions, respectively, and note that any poverty index satisfying (5) is continuous on $\mathscr{D}_{p}$. Hence, by Proposition 1 applied to this smaller domain, there exist functions $\phi: D_{p} \rightarrow \mathbb{R}$ and $F: \phi\left(D_{p}\right) \rightarrow \mathbb{R}$ such that

$$
P(x)=F\left[\frac{1}{n(x)} \sum_{i=1}^{n(x)} \phi\left(x_{i}\right)\right] \quad \text { for all } x \in \mathscr{D}_{p},
$$

\footnotetext{
${ }^{12}$ This means, for example, that for each subgroup consistent index $C_{\beta}$ of Clark et al. there must be a corresponding canonical index. In fact, the family of decomposable indices of Chakravarty (1983a), suitably extended, will do the trick.
} 
where $F$ is continuous and increasing; $\phi$ is continuous and nonincreasing; and $\phi(t)=0$ for $t=z$. Extending $\phi$ to the domain $D$ by setting $\phi(t)=0$ for $t \geq z$ allows us to construct the canonical index $P^{\phi}$ associated with $\phi$, and to write

$$
P(x)=F\left[P^{\phi}(x)\right] \quad \text { for all } x \in \mathscr{D}_{p} .
$$

Now consider any distribution $y \in \mathscr{D}_{r}$ and any distributions $w, w^{\prime} \in \mathscr{D}_{p}$ for which $n(w)=n\left(w^{\prime}\right)$. Subgroup consistency implies

$$
P(w, y) \geq P\left(w^{\prime}, y\right) \quad \text { if and only if } \quad P^{\phi}(w) \geq P^{\phi}\left(w^{\prime}\right) .
$$

Hence there exists some function $\Pi_{1}$, increasing in its first argument, such that

$$
P(w, y)=\Pi_{1}\left(P^{\phi}(w), n(w), y\right) .
$$

The focus axiom implies $P(w, y)=P\left(w, y^{\prime}\right)$ whenever $y, y^{\prime} \in \mathscr{D}_{r}$ and $n(y)=$ $n\left(y^{\prime}\right)$. So (29) may be rewritten as

$$
P(w, y)=\Pi_{2}\left[P^{\phi}(w), n(w), n(y)\right]
$$

or, since $H(w, y)=n(w) /[n(w)+n(y)]$ and $P^{\phi}(w, y)=H(w, y) P^{\phi}(w)$, as

$$
P(w, y)=\Pi_{3}\left[P^{\phi}(w, y), H(w, y), n(y)\right] .
$$

Replication invariance ensures $\Pi_{3}\left[P^{\phi}, H, n\right]=\Pi_{3}\left[P^{\phi}, H, k n\right]$ for all positive integers $k$ and $n$, which enables (30) to be further simplified to

$$
P(x)=\pi\left[P^{\phi}(x), H(x)\right]
$$

for all $x \in \mathscr{D}_{p} \times \mathscr{D}_{r}$, where $\pi\left[P^{\phi}, H\right]$ is increasing in $P^{\phi}$. Note that $P^{\phi}$ and $H$ are both zero on $\mathscr{D}_{r}$, while by (2) and (4) $P$ is some constant value on $\mathscr{D}_{r}$. So we may extend (31) to hold for all $x \in \mathscr{D}$ by defining $\pi[0,0]$ to be this constant value on $\mathscr{D}_{r}$ and setting $\pi\left[P^{\phi}(x), H(x)\right]=F\left(P^{\phi}(x)\right)$ for $x \in \mathscr{D}_{p}$. Then $\pi\left[P^{\phi}, H\right]$ is increasing in $P^{\phi} ; \pi$ is continuous in $P^{\phi}$ by (5); and since an increment to a poor person just on the poverty line lowers $H$ and leaves $P^{\phi}$ unchanged, $\pi$ must be nondecreasing in $H$ by (3). Thus we obtain the following proposition:

Proposition 2: Let $P: \mathscr{D} \rightarrow \mathbb{R}$ be a subgroup consistent poverty index. Then there exist real valued functions $\phi$ and $\pi$ such that

$$
P(x)=\pi\left[P^{\phi}(x), H(x)\right] \text { for all } x \in \mathscr{D},
$$

where $P^{\phi}$ is the canonical index associated with $\phi ; \phi$ satisfies the properties given in (23); and $\pi\left(P^{\phi}, H\right)$ is continuous and increasing in $P^{\phi}$, and nondecreasing in $H$.

Proposition 2 shows that any subgroup consistent index $P$ is some function of $H$ and $P^{\phi}$. We now determine the forms this function may take. One possibility, mentioned earlier, is that $P$ is a transformation of a convex combination of $H$ and $P^{\phi}$. The second possibility requires a new definition. We say that $Q$ is a lexical combination of $H$ and $P^{\phi}$ if

$$
\begin{array}{ll}
Q(x) \geq Q(y) \text { if and only if } & H(x)>H(y) \text { or } \\
& {\left[H(x)=H(y) \text { and } P^{\phi}(x) \geq P^{\phi}(y)\right] .}
\end{array}
$$


A lexical combination places the headcount ratio first in poverty considerations and only consults the index $P^{\phi}$ in the case of a tie. It may be confirmed that any such index $Q$ must be subgroup consistent. ${ }^{13}$ Our next result shows that these two methods of combining $H$ and $P^{\phi}$ are the only ways of forming a subgroup consistent index. More precisely, the class of subgroup consistent poverty indices is completely characterized as follows:

Proposition 3: $P: \mathscr{D} \rightarrow \mathbb{R}$ is a subgroup consistent poverty index if and only if there exists a canonical index $P^{\phi}$ such that either

$$
P(x)=F\left[P^{\phi}(x)\right] \quad \text { for all } x \in \mathscr{D}
$$

for some continuous, increasing transformation $F$; or

$$
P(x)=F\left[\lambda H(x)+(1-\lambda) P^{\phi}(x)\right] \quad \text { for all } x \in \mathscr{D}
$$

for some $\lambda \in(0,1)$ and some increasing transformation $F(u)$, continuous for $u>0$; or

$$
P \text { is a lexical combination of } H \text { and } P^{\phi} \text {, continuous for fixed } H \text {. }
$$

In other words, $P$ is essentially a convex combination or a lexical combination of $H$ and $P^{\phi}$. The proof of Proposition 3 is rather lengthy and is relegated to the Appendix. However, the intuition is straightforward. The key consideration is whether the function $\pi\left[P^{\phi}, H\right]$ in Proposition 2 allows a reduction in $P^{\phi}$ to compensate for a higher value of $H$. If no trade-off of this type is permitted, a lexical index (33c) results. Otherwise $P$ can be shown to be either an increasing transformation of $P^{\phi}$ as indicated in (33a) or a convex combination of the form described in (33b).

The result contained in Proposition 3 may be restated in a slightly different way by noting that the expressior $\lambda H(x)+(1-\lambda) P^{\phi}(x)$ in (33b) may be rewritten as

$$
P^{d}(x):=\frac{1}{n(x)} \sum_{i=1}^{n(x)} d\left(x_{i}\right)
$$

where $d(t)=\lambda+(1-\lambda) \phi(t)$ for $t \leq z$ and $d(t)=(1-\lambda) \phi(t)=0$ for $t>z$. The function $d$ is a generalization of our earlier function $\phi$, and supports the same interpretation as a measure of individual deprivation. It also inherits the same properties, apart from a possible discontinuity at $z$, so that

$$
\begin{aligned}
& d: D \rightarrow \mathbb{R} \text { is nonincreasing; } d(t) \text { is continuous for } t \leq z ; \text { and } \\
& d(t)=0 \text { for } t>z \text {. }
\end{aligned}
$$

\footnotetext{
${ }^{13}$ Since $H$ can take only rational values, it is clear that such indices exist for any given $P^{\phi}$. The existence of a representation of this lexicographic ordering, when $\phi(D) \neq\{0\}$, is equivalent to the existence of an increasing function on $(0,1]$ that is discontinuous at each rational. An example of the latter function may be found in Gelbaum and Olmsted (1964, p. 28). We thank Bill Novshek for this reference.
} 
If we now expand the set of canonical indices to cover any index $P^{d}$ corresponding to some function $d$ satisfying (35), Corollary 3 follows from Proposition 3:

CoRollary 3: If $P$ is a subgroup consistent poverty index then there exists a canonical index $P^{d}$ such that either $P$ is an increasing transformation of $P^{d}$ or else $P$ is a lexical combination of $H$ and $P^{d}$.

Notice that any $P^{d}$ satisfying (34) is clearly decomposable. Furthermore, we have the following corollary, as we show in the Appendix:

Corollary 4: $P$ is a decomposable poverty index if and only if $P=P^{d}+c$ for some canonical index $P^{d}$ and some constant $c$.

So when the continuity property is relaxed, we are still able to derive close analogues of both Corollary 1 and Corollary 2 . The only significant change is due to the fact that the noncontinuous case admits lexical forms of indices.

\section{RELATIVE AND ABSOLUTE POVERTY INDICES}

In the previous section we characterized subgroup consistent poverty indices under the maintained assumption of a fixed and given poverty line. When variations in the poverty line are explicitly taken into account, our results take on a slightly different form. For example, equation (23) from Proposition 1 becomes

$$
P(x ; z)=F\left[\frac{1}{n(x)} \sum_{i=1}^{n(x)} \phi\left(x_{i} ; z\right), z\right] \text { for all } x \in \mathscr{D} \text { and } z \in D
$$

where, at each specific $z$, the functions $F(., z)$ and $\phi(. ; z)$ have the properties listed in Proposition 1. Since the way that $F$ and $\phi$ depend on $z$ is left unspecified, $P$ may alter substantially at different poverty lines. For example, $P$ could well be the headcount ratio $H$ at some $z$, and the per-capita income gap $G$ at some other level $z^{\prime}$. To ensure that a poverty index exhibits a reasonable degree of coherence we may wish to consider a further type of property that coordinates $P$ at different poverty lines. This is typically accomplished by requiring the poverty value to be invariant to certain kinds of simultaneous changes in incomes and the poverty line. Blackorby and Donaldson (1980) consider two kinds of invariance properties, leading to classes of poverty indices they call "relative" and "absolute." Relative indices are invariant to changes in scale, such as a doubling of the poverty line and all incomes, while absolute indices are invariant to translations or additions of the same absolute amount to each income and to the poverty line. To express these requirements rigorously, we need to state a few more definitions.

We say that $\left(x^{\prime} ; z^{\prime}\right) \in \mathscr{D} \times D$ is obtained from $(x ; z) \in \mathscr{D} \times D$ by a relative change if $\left(x^{\prime} ; z^{\prime}\right)=\lambda(x ; z)$ for some $\lambda>0$; and by an absolute change if $\left(x^{\prime} ; z^{\prime}\right)=(x ; z)+(\lambda \mathbf{1} ; \lambda)$ for some $\lambda>0$, where $\mathbf{1}$ is an appropriate sized vector 
of 1's. The two invariance properties may then be defined as follows:

Scale Invariance: $P\left(x^{\prime} ; z^{\prime}\right)=P(x ; z)$ whenever $\left(x^{\prime} ; z^{\prime}\right) \in \mathscr{D} \times D$ is obtained from $(x ; z) \in \mathscr{D} \times D$ by a relative change.

Translation Invariance: $P\left(x^{\prime} ; z^{\prime}\right)=P(x ; z)$ whenever $\left(x^{\prime} ; z^{\prime}\right) \in \mathscr{D} \times D$ is obtained from $(x ; z) \in \mathscr{D} \times D$ by an absolute change.

Following Blackorby and Donaldson, relative poverty indices are those that are scale invariant, while absolute poverty indices are translation invariant.

For simplicity, we restrict attention to the domain $\bar{D}=\mathbb{R}_{++}$, and the associated set of distributions $\overline{\mathscr{D}}$, for the remainder of this section. We first identify the set of all subgroup consistent, relative poverty indices satisfying continuity. For any relative poverty index $P$, we must have $P(x ; z)=P(x / z ; 1)$ for all $(x ; z) \in \bar{D} \times \bar{D}$. Setting $\phi(t):=\phi(t ; 1)$ and $F_{R}(u):=F(u, 1)$, and substituting into (36), it follows immediately that:

Proposition 4: $P_{R}: \overline{\mathscr{D}} \rightarrow \mathbb{R}$ is a continuous, subgroup consistent, relative poverty index if and only if there exist $\phi: \bar{D} \rightarrow \mathbb{R}$ and $F_{R}: \phi(\bar{D}) \rightarrow \mathbb{R}$ such that

$$
P_{R}(x ; z)=F_{R}\left[\frac{1}{n(x)} \sum_{i=1}^{n(x)} \phi\left(x_{i} / z\right)\right] \quad \text { for all } x \in \overline{\mathscr{D}} \text { and } z \in \bar{D},
$$

where $F_{R}$ is continuous and increasing; $\phi$ is continuous and nonincreasing; and $\phi(t)=0$ for all $t \geq 1$.

We next consider the subset of indices of the form (36) that are absolute poverty indices. Since $P$ must be translation invariant, for any $x \in \bar{D}^{2}$ we have

$$
P\left(x_{1}+t, x_{2}+t ; z+t\right)=P\left(x_{1}, x_{2} ; z\right) \quad \text { for all } z \in \bar{D} \text { and } t \geqslant 0,
$$

and hence

$$
F\left[\frac{1}{2} \sum_{i=1}^{2} \phi\left(x_{i}+t ; z+t\right), z+t\right]=F\left[\frac{1}{2} \sum_{i=1}^{2} \phi\left(x_{i} ; z\right), z\right] .
$$

Setting $x_{1}=x_{2}=v$ then yields

$$
F[\phi(v+t ; z+t), z+t]=F[\phi(v ; z), z] \text { for all } z, v \in \bar{D} \text { and } t \geq 0,
$$

and the properties of $F$ allow us to write

$$
\phi(v+t ; z+t)=G_{z t}[\phi(v ; z)],
$$

where $G_{z t}(u):=F^{-1}[F(u, z), z+t]$ is continuous and increasing for $u \in \phi(\bar{D} ; z)$, and $G_{z t}(0)=0$. Substituting (40) into (38), and defining $u_{i}:=\phi\left(x_{i} ; z\right)$ for $i=1,2$, 
we obtain

$$
\begin{aligned}
G_{z t}\left[\frac{1}{2}\left(u_{1}+u_{2}\right)\right] & =F^{-1}\left[F\left[\frac{1}{2} \phi\left(x_{1} ; z\right)+\frac{1}{2} \phi\left(x_{2} ; z\right), z\right], z+t\right] \\
& =\frac{1}{2} \phi\left(x_{1}+t ; z+t\right)+\frac{1}{2} \phi\left(x_{2}+t ; z+t\right) \\
& =\frac{1}{2} G_{z t}\left(u_{1}\right)+\frac{1}{2} G_{z t}\left(u_{2}\right)
\end{aligned}
$$

where $G_{z t}(0)=0$. The solution of this Jensen equation (Aczel (1966, p. 46)) yields $G_{z t}(u)=r(z, z+t) u$ for some positive function $r$, and hence

$$
\phi(v+t ; z+t)=r(z, z+t) \phi(v ; z) \quad \text { for all } z, v \in \bar{D} \text { and } t \geq 0 .
$$

Setting $\zeta(\tau ; z):=\phi(z-\tau ; z)$ for $z \in \bar{D}$ and $\tau \leq z$, we obtain the Sincov equation

$$
\zeta(z-v ; z+t)=r(z, z+t) \zeta(z-v ; z),
$$

whose solution (Aczel (1966, p. 303)) implies

$$
\begin{aligned}
& \zeta(\tau ; z)=s(z) \psi(\tau)=\phi(z-\tau ; z), \\
& r(z, z+t)=s(z+t) / s(z),
\end{aligned}
$$

for some functions $s: \bar{D} \rightarrow \mathbb{R}$ and $\psi: \mathbb{R} \rightarrow \mathbb{R}$. Since $r(.,$.$) is positive, we may$ assume $s(z)>0$ for all $z \in \bar{D}$. From (44) and the properties of $\phi$, it then follows that $\psi$ is continuous and nondecreasing; and that $\psi(\tau)=0$ for $\tau \leq 0$.

Returning to (39) we now deduce that

$$
F\left[s\left(z^{\prime}\right) \psi(\tau), z^{\prime}\right]=F[s(z) \psi(\tau), z]
$$

for all $z, z^{\prime} \in \bar{D}$ and $\tau \in \mathbb{R}$ such that $\tau<z \leq z^{\prime}$. Clearly (45) also holds if $\tau<z^{\prime} \leq z$. Now defining $h: \mathbb{R} \rightarrow \mathbb{R}$ by

$$
h(\tau):= \begin{cases}F[s(\tau+1) \psi(\tau), \tau+1] & \text { for } \tau \geq 0 \\ F[0,1] & \text { for } \tau \leq 0\end{cases}
$$

and setting $z^{\prime}=\tau+1$ when $\tau \geq 0$ and $z^{\prime}=1$ when $\tau \leq 0$, we obtain

$$
h(\tau)=F[s(z) \psi(\tau), z] \quad \text { for all } z \in \bar{D} \text { and } \tau<z .
$$

But since $s(z)>0$ and since $F[u, z]$ is continuous and increasing in $u$, it follows that $h(\tau)$ is a contınuous and increasing function of $\psi(\tau)$. Hence there exists some continuous, increasing function $F_{A}: \psi(\mathbb{R}) \rightarrow \mathbb{R}$ such that

$$
F_{A}[\psi(z-v)]=h(z-v)=F[s(z) \psi(z-v), z] \text { for all } z, v \in \bar{D} \text {. }
$$

Finally, choose any $x \in \overline{\mathscr{D}}$ and any $z \in \bar{D}$, and define

$$
\xi=\frac{1}{n(x)} \sum_{i=1}^{n(x)} \psi\left(z-x_{i}\right)
$$

noting that $\xi \in \psi(\mathbb{R})$, since $\psi(\mathbb{R})$ is a convex set. Combining (36), (44), and (47) 
then yields

$$
\begin{aligned}
P(x ; z) & =F\left[\frac{1}{n(x)} \sum_{i=1}^{n(x)} \phi\left(x_{i} ; z\right), z\right]=F\left[s(z) \frac{1}{n(x)} \sum_{i=1}^{n(x)} \psi\left(z-x_{i}\right), z\right] \\
& =F[s(z) \xi, z]=F_{A}[\xi]
\end{aligned}
$$

and establishes the following proposition:

Proposition 5: $P_{A}: \bar{D} \rightarrow \mathbb{R}$ is a continuous, subgroup consistent, absolute poverty index if and only if there exist $\psi: \mathbb{R} \rightarrow \mathbb{R}$ and $F_{A}: \psi(\mathbb{R}) \rightarrow \mathbb{R}$ such that

$$
P_{A}(x ; z)=F_{A}\left[\frac{1}{n(x)} \sum_{i=1}^{n(x)} \psi\left(z-x_{i}\right)\right] \text { for all } x \in \bar{D} \text { and } z \in \bar{D},
$$

where $F_{A}$ is continuous and increasing; $\psi$ is continuous and nondecreasing; and $\psi(\tau)=0$ for all $\tau \leq 0$.

An obvious question to ask at this stage is whether a subgroup consistent index can be both a relative and an absolute index of poverty. To answer this question we observe that if the poverty index $P: \overline{\mathscr{D}} \rightarrow \mathbb{R}$ is both scale and translation invariant, then for any poverty line $z \in \bar{D}$ and all one-person distributions $v, v^{\prime} \in \bar{D}$ satisfying $v<v^{\prime}<z$ we have

$$
\begin{aligned}
P(v ; z) & =P\left(v\left(z-v^{\prime}\right) ; z\left(z-v^{\prime}\right)\right) \\
& =P\left(v\left(z-v^{\prime}\right)+z\left(v^{\prime}-v\right) ; z\left(z-v^{\prime}\right)+z\left(v^{\prime}-v\right)\right) \\
& =P\left(v^{\prime}(z-v) ; z(z-v)\right)=P\left(v^{\prime} ; z\right) .
\end{aligned}
$$

From Proposition 2 we deduce that $\phi(v ; z)=\phi\left(v^{\prime} ; z\right)$ for all $v<v^{\prime}<z$, and hence, by the properties of $\phi(. ; z)$,

$$
\phi(v ; z)=\phi(z ; z)=0 \quad \text { for all } v, z \in \bar{D} .
$$

Now if $P$ is also continuous, it follows that $\phi(t):=\phi(t ; 1)=0$ for all $t \in \bar{D}$, and hence, by Proposition $4, P$ is a trivial poverty index. The set of possibilities does not become much larger when continuity is relaxed. For from (52) we deduce that $P^{\phi}(x ; z)=0$ for all $x \in \bar{D}$ and $z \in \bar{D}$. Proposition 2 then implies

$$
P(x ; z)=\pi[0, H(x ; z) ; z]
$$

and, since $P$ and $H$ are both scale invariant, we obtain

$$
P(x ; z)=P(x / z ; 1)=\pi[0, H(x ; z) ; 1] .
$$

So $P$ is a nondecreasing transformation of the headcount ratio. Furthermore, by appealing to Proposition 3, and noting that $P$ is trivial if $P^{\phi} \equiv 0$ in (33a), we are able to state the following:

Proposition 6: Let $P: \bar{D} \rightarrow \mathbb{R}$ be a nontrivial poverty index. Then $P$ is subgroup consistent and both relative and absolute if and only if $P$ is an increasing transformation of the headcount ratio. 
Since the requirement that an index be both relative and absolute is clearly very restrictive, we might instead ask whether a pair of relative and absolute poverty indices can be compatible, in the sense that any change in the income distribution which raises the level of poverty for the relative index also increases the absolute poverty value. Formally, we define compatibility as follows:

Definition: The poverty indices $P$ and $P^{\prime}$ are compatible if for all $z \in D$ and $x, y \in \mathscr{D}$,

$$
P(x ; z) \geq P(y ; z) \quad \text { if and only if } \quad P^{\prime}(x ; z) \geq P^{\prime}(y ; z) .
$$

For continuous indices, the definition of compatibility implies

$$
P^{\prime}(x ; z)=\bar{\Gamma}[P(x ; z), z],
$$

for some function $\bar{\Gamma}(P, z)$ continuous and increasing in $P$. So if the relative poverty index $P_{R}$ given by (37) and the absolute poverty index $P_{A}$ given by (50) are compatible, it follows from (56) that

$$
\frac{1}{2} \sum_{i=1}^{2} \psi\left(z-x_{i}\right)=\Gamma\left[\frac{1}{2} \sum_{i=1}^{2} \phi\left(x_{i} / z\right), z\right] \text { for all } x_{1}, x_{2}, z \in \bar{D},
$$

for some function $\Gamma[u, z]$, continuous and increasing in $u$, and satisfying $\Gamma[0, z]=0$.

We now examine the implications of (57) for the functions $\psi$ and $\phi$. Setting $x=(v, v)$ gives

$$
\psi(z-v)=\Gamma[\phi(v / z), z] \quad \text { for all } v, z \in \bar{D} .
$$

Substitution into (57) then yields the Jensen equation

$$
\frac{1}{2} \sum_{i=1}^{2} \Gamma\left[\phi\left(x_{i} / z\right), z\right]=\Gamma\left[\frac{1}{2} \sum_{i=1}^{2} \phi\left(x_{i} / z\right), z\right],
$$

whose solution is $\Gamma(u, z)=c(z) u$, for some positive function $c: \bar{D} \rightarrow \mathbb{R}$. Hence,

$$
\psi(z-v)=c(z) \phi(v / z) \text { for all } v, z \in \bar{D},
$$

which in turn implies

$$
\psi(z \theta)=c(z) \phi(1-\theta) \text { for all } z \in \bar{D} \text { and } \theta \in[0,1) .
$$

Equation (61) is a Pexider equation whose solution (Aczel (1966, p. 145)) implies that there exist constants $\eta_{1}, \eta_{2}$, and $\alpha$ such that $\psi(\tau)=\eta_{1} \tau^{\alpha}$ for $\tau>0$ and $\phi(\theta)=\eta_{2}(1-\theta)^{\alpha}$ for $\theta \in(0,1]$. Since $\psi(\tau)$ and $\phi(\theta)$ are nonnegative, and $\psi$ is nondecreasing, we deduce that $\eta_{1}, \eta_{2}$, and $\alpha$ are all nonnegative. Furthermore, $\eta_{1} \neq 0$ and $\eta_{2} \neq 0$ by nontriviality, and $\alpha \neq 0$ by continuity of $\psi$ and $\phi$. Substituting into (37) and (50), and recalling the Foster et al. (1984) class of indices $P_{\alpha}$ given in (9), we therefore obtain the following Proposition:

Proposition 7: The relative poverty index $P_{R}: \overline{\mathscr{D}} \rightarrow \mathbb{R}$ and the absolute poverty index $P_{A}: \overline{\mathscr{D}} \rightarrow \mathbb{R}$ are nontrivial, continuous, subgroup consistent and compatible if 
and only if there is some $\alpha>0$ such that $P_{R}$ is a continuous increasing transformation of $P_{\alpha}$, and $P_{A}$ is a continuous increasing transformation of $z^{\alpha} P_{\alpha}$.

This final result may be viewed as a complete axiomatic characterization of the Foster et al. class of poverty indices. It establishes that the set $\left\{\lambda P_{\alpha} \mid \alpha>0\right.$; $\lambda \geq 0$ \} contains the canonical forms of all continuous, subgroup consistent, relative poverty indices which are compatible with some continuous, subgroup consistent, absolute poverty index. If we choose to adopt a nontrivial relative measure of poverty which is not an increasing function of one of these $P_{\alpha}$, then the chosen index must either violate continuity or subgroup consistency, or else have no absolute poverty index counterpart which ranks distributions in the same way for any given poverty line.

\section{CONCLUDING REMARKS}

This paper has examined a property of poverty indices-subgroup consistency - which ensures that overall poverty does not respond perversely to changes in poverty levels within population subgroups. Our objective has been to identify the specific implications of subgroup consistency, and for that reason we have deliberately kept the other properties of poverty indices to a minimum. As a consequence, we have refrained from discussing a number of further restrictions that are frequently imposed: for example, the normalization condition which says that the poverty level is zero when all incomes are above the poverty line; the strict monotonicity requirement that poverty decreases whenever the income of someone below the poverty line is raised; and the transfer axiom, which states that poverty declines when a progressive transfer takes place between two poor people. Fortunately, the form of our results, and in particular the additive structure of the "canonical" indices $P^{\phi}$ and $P^{d}$, make it very easy to incorporate these and other additional restrictions. For instance, the normalization axiom implies that $F(0)=0$ in Proposition 1 and that $c=0$ in Corollary 1 . Given the form of the subgroup consistent poverty index $P$ described in (33), we may also deduce that $P$ is strictly monotonic if and only if the individual deprivation function $\phi(t)$ is decreasing for $t<z$; and that $P$ satisfies the transfer axiom if and only if $\phi(t)$ is strictly convex for $t \leq z$. Notice that either strict monotonicity or the transfer axiom is sufficient to rule out the headcount ratio.

Our results may also be extended by considering alternative formulations of the subgroup consistency property. One possibility is to suppose that overall poverty does not decrease (rather than increases) when the poverty level rises in one subgroup and remains the same elsewhere. This "weak subgroup consistency" condition has the effect of replacing (6a) with the requirement $P(x, y ; z)$ $\geq P\left(x^{\prime}, y^{\prime} ; z\right)$, and implies that $P$ must be "weakly separable" in the sense of Mak (1986). It admits a number of poverty indices which are not encompassed by the forms described in Proposition 3, the most interesting examples being the 
relative index

$$
P(x ; z)=1-\frac{\min \left\{x_{1}, \ldots, x_{n(x)}, z\right\}}{z}
$$

and its compatible absolute index

$$
P(x ; z)=z-\min \left\{x_{1}, \ldots, x_{n(x)}, z\right\} .
$$

Each of these indices focuses on the lowest income in the population, and in that respect provides a "Rawlsian" type of poverty measure. Neither satisfies subgroup consistency (6), since a rise in the poverty level of one subgroup will not increase the overall poverty value unless that subgroup contains the poorest member of society. Clearly, the class of admissible indices expands if our subgroup consistency property is replaced with this weaker variant. But the precise implications of weak subgroup consistency remains a topic for future investigation.

Department of Economics and Business Administration, Vanderbilt University, Nashville, TN 37235, U.S.A.

\section{and}

Department of Economics, University of Essex, Wivenhoe Park, Colchester C04 $35 Q$, England

Manuscript received August, 1988; revision received April, 1990.

\section{APPENDIX}

To prove Proposition 3 and Corollary 4, we consider any subgroup consistent poverty index $P$ and recall that Proposition 2 allows $P$ to be written in the form

$$
P(x)=\pi\left[P^{\phi}(x), H(x)\right]
$$

where $P^{\phi}$ is some canonical index; $H$ is the headcount ratio; and $\pi\left[P^{\phi}, H\right]$ is continuous and increasing in $P^{\phi}$, and nondecreasing in $H$. Note that $\pi\left[P^{\phi}, H\right]$ is defined for all elements $\left(P^{\phi}, H\right)$ in the set

$$
\mathcal{P}:=\left\{\left(P^{\phi}(x), H(x)\right), x \in \mathscr{D}\right\}=\left\{\left(P^{\phi}, H\right) \mid H \in \mathscr{H} ; P^{\phi} \in H \cdot \phi(D)\right\},
$$

where $\mathscr{H}$ denotes the set of rationals in $[0,1]$. We begin by establishing the conditions which $\pi$ must satisfy if $P$ is subgroup consistent or decomposable.

Consider any $\left(P_{k}, H_{k}\right),\left(P_{l}, H_{j}\right)$ and $\left(P_{l}, H_{l}\right) \in \mathscr{P}$. Let $x^{k} \in \mathscr{D}$ satisfy $P^{\phi}\left(x^{k}\right)=P_{k}$ and $H\left(x^{k}\right)=$ $H_{k}$, and choose $x^{j}$ and $x^{l}$ in a similar way. Since $P^{\phi}$ and $H$ are replication invariant, we may assume $n\left(x^{k}\right)=n\left(x^{\jmath}\right)=n\left(x^{l}\right)$. Let $x^{k}(r)$ denote the $r$-replication of $x^{k}$, and let $m$ and $p$ be any positive integers. Then, by replication invariance,

$$
P\left(x^{\jmath}\right)<P\left(x^{k}\right) \text { if and only if } P\left(x^{\jmath}(m)\right)<P\left(x^{k}(m)\right)
$$

and by subgroup consistency

$$
P\left(x^{j}(m)\right)<P\left(x^{k}(m)\right) \text { if and only if } P\left(x^{j}(m), x^{l}(p)\right)<P\left(x^{k}(m), x^{l}(p)\right) .
$$


Since $P^{\phi}$ and $H$ are decomposable, we know that

$$
\begin{aligned}
& P^{\phi}\left(x^{k}(m), x^{l}(p)\right)=\theta P^{\phi}\left(x^{k}\right)+(1-\theta) P^{\phi}\left(x^{l}\right)=\theta P_{k}+(1-\theta) P_{l} \\
& H\left(x^{k}(m), x^{l}(p)\right)=\theta H\left(x^{k}\right)+(1-\theta) H\left(x^{l}\right)=\theta H_{k}+(1-\theta) H_{l},
\end{aligned}
$$

where $\theta=m /(m+p)$. So combining (A1)-(A6) yields

$$
\begin{aligned}
& \pi\left[P_{j}, H_{j}\right] \leq \pi\left[P_{k}, H_{k}\right] \quad \text { if and only if } \\
& \pi\left[\theta P_{j}+(1-\theta) P_{l}, \theta H_{j}+(1-\theta) H_{l}\right] \leq \pi\left[\theta P_{k}+(1-\theta) P_{l}, \theta H_{k}+(1-\theta) H_{l}\right]
\end{aligned}
$$

for any $\left(P_{k}, H_{k}\right),\left(P_{l}, H_{l}\right),\left(P_{l}, H_{l}\right) \in \mathscr{A}$ and any rational $\theta \in(0,1)$. Furthermore, if $P$ is also decomposable, then using (7), (A1), (A5), and (A6) we deduce

$$
\pi\left[\theta P_{j}+(1-\theta) P_{k}, \theta H_{j}+(1-\theta) H_{k}\right]=\theta \pi\left[P_{J}, H_{J}\right]+(1-\theta) \pi\left[P_{k}, H_{k}\right]
$$

for all $\left(P_{j}, H_{j}\right),\left(P_{k}, H_{k}\right) \in \mathscr{S}$ and any $\theta \in \mathscr{H}$.

Proof of Proposition 3: We will distinguish two cases depending on whether the statement:

$$
\text { for } x, x^{\prime} \in \mathscr{D}: P(x)<P\left(x^{\prime}\right) \text { whenever } H(x)<H\left(x^{\prime}\right) \text {, }
$$

is true or false. Suppose first that (A9) is true. Then since $\pi\left[P^{\phi}, H\right]$ is increasing in $P^{\phi}$, we also know that:

(A10) for $x, x^{\prime} \in \mathscr{D}: P(x) \leq P\left(x^{\prime}\right)$ whenever $H(x)=H\left(x^{\prime}\right)$ and $P^{\phi}(x) \leq P^{\phi}\left(x^{\prime}\right)$.

It follows immediately that $P(x)$ is a lexical combination of $H(x)$ and $P^{\phi}(x)$, as indicated in (33c). Notice that this degenerates to an increasing transformation of $H(x)$ alone if $\phi(D)=\{0\}$. and

Alternatively, suppose (A9) is false. Then there exist $\left(P_{1}, H_{1}\right),\left(P_{2}^{\prime}, H_{2}\right) \in \mathscr{f}$ such that $H_{1}<H_{2}$

(A11) $\quad \pi\left[P_{1}, H_{1}\right] \geq \pi\left[P_{2}^{\prime}, H_{2}\right]$.

As $\pi\left[P^{\phi}, H\right]$ is nondecreasing in $H$, it follows that

$$
\pi\left[P_{1}, H_{2}\right] \geq \pi\left[P_{1}, H_{1}\right] \geq \pi\left[P_{2}^{\prime}, H_{2}\right],
$$

which implies $P_{1} \geq P_{2}^{\prime}$, since $\pi\left[P^{\phi}, H\right]$ is increasing in $P^{\phi}$. Furthermore, since $\pi\left[P^{\phi}, H\right]$ is continuous in $P^{\phi}$, there is some $P_{2} \in\left[P_{2}^{\prime}, P_{1}\right]$ such that $\pi\left[P_{1}, H_{1}\right]=\pi\left[P_{2}, H_{2}\right]$. Hence we conclude that there exist $\left(P_{1}, H_{1}\right),\left(P_{2}, H_{2}\right) \in \mathscr{f}$ such that

$$
\pi\left[P_{1}, H_{1}\right]=\pi\left[P_{2}, H_{2}\right] ; \quad H_{1}<H_{2} ; \quad \text { and } P_{2} \leq P_{1} .
$$

Let $\lambda:=\left(P_{1}-P_{2}\right) /\left(P_{1}-P_{2}+H_{2}-H_{1}\right) \in[0,1)$, let $g\left[P^{\phi}, H\right]:=\lambda H+(1-\lambda) P^{\phi}$ for $\left(P^{\phi}, H\right) \in \mathcal{P}$, and consider any $\left(P_{3}, H_{3}\right),\left(P_{4}, H_{4}\right) \in \mathcal{S}$. We now proceed to establish

$$
\pi\left[P_{3}, H_{3}\right] \gtrless \pi\left[P_{4}, H_{4}\right] \text { if and only if } g\left[P_{3}, H_{3}\right] \gtrless g\left[P_{4}, H_{4}\right] .
$$

Given that $\left(P_{3}, H_{3}\right)$ and $\left(P_{4}, H_{4}\right)$ are treated symmetrically in (A14), we may assume that $H_{3} \leq H_{4}$. Furthermore, since both $\pi\left[P^{\phi}, H\right]$ and $g\left[P^{\phi}, H\right]$ are increasing in $P^{\phi}$, statement (A14) is clearly true when $H_{3}=H_{4}$. Therefore, we need deal only with the case $H_{3}<H_{4}$.

By setting $j=3, k=4, l=1$, and $\theta=\theta^{*}:=\left(H_{2}-H_{1}\right) /\left(H_{2}-H_{1}+H_{4}-H_{3}\right) \in(0,1)$ in (A7), we obtain

$$
\begin{aligned}
& \pi\left[P_{3}, H_{3}\right] \gtrless \pi\left[P_{4}, H_{4}\right] \text { if and only if } \\
& \begin{aligned}
& \pi\left[\theta^{*} P_{3}+\left(1-\theta^{*}\right) P_{1}, \theta^{*} H_{3}+\left(1-\theta^{*}\right) H_{1}\right] \\
& \gtrless \pi\left[\theta^{*} P_{4}+\left(1-\theta^{*}\right) P_{1}, \theta^{*} H_{4}+\left(1-\theta^{*}\right) H_{1}\right] .
\end{aligned}
\end{aligned}
$$

In addition, by setting $j=1, k=2, l=3$, and $\theta=\left(1-\theta^{*}\right)$, we deduce from (A7) and (A13) that

$$
\begin{aligned}
& \pi\left[\left(1-\theta^{*}\right) P_{2}+\theta^{*} P_{3},\left(1-\theta^{*}\right) H_{2}+\theta^{*} H_{3}\right] \\
& \quad=\pi\left[\left(1-\theta^{*}\right) P_{1}+\theta^{*} P_{3},\left(1-\theta^{*}\right) H_{1}+\theta^{*} H_{3}\right] .
\end{aligned}
$$


Therefore, by combining (A15) and (A16), and noting that $H^{*}:=\theta^{*} H_{4}+\left(1-\theta^{*}\right) H_{1}=\theta^{*} H_{3}+$ $\left(1-\theta^{*}\right) H_{2}$, we have $\pi\left[P_{3}, H_{3}\right] \gtrless \pi\left[P_{4}, H_{4}\right]$ if and only if

$$
\pi\left[\left(1-\theta^{*}\right) P_{2}+\theta^{*} P_{3}, H^{*}\right] \gtrless \pi\left[\theta^{*} P_{4}+\left(1-\theta^{*}\right) P_{1}, H^{*}\right],
$$

or, equivalently, since $\pi\left[P^{\phi}, H\right]$ is increasing in $P^{\phi}$,

$$
\left(1-\theta^{*}\right) P_{2}+\theta^{*} P_{3} \gtrless \theta^{*} P_{4}+\left(1-\theta^{*}\right) P_{1} \text {. }
$$

Since (A18) is in turn equivalent to the statement

$$
P_{3}-P_{4} \gtrless \frac{\left(1-\theta^{*}\right)}{\theta^{*}}\left(P_{1}-P_{2}\right)=\frac{H_{4}-H_{3}}{H_{2}-H_{1}}\left(P_{1}-P_{2}\right)=\frac{\lambda}{(1-\lambda)}\left(H_{4}-H_{3}\right) \text {, }
$$

and hence also

$$
g\left[P_{3}, H_{3}\right]=\lambda H_{3}+(1-\lambda) P_{3} \gtrless \lambda H_{4}+(1-\lambda) P_{4}=g\left[P_{4}, H_{4}\right],
$$

it follows that (A14) is true for all $\left(P_{3}, H_{3}\right),\left(P_{4}, H_{4}\right) \in \mathscr{f}$.

From (A14) we conclude that there is some $\lambda \in[0,1)$ and some increasing function $F$ such that

$$
P(x)=\pi\left[P^{\phi}(x), H(x)\right]=F\left[\lambda H(x)+(1-\lambda) P^{\phi}(x)\right] \text { for all } x \in \mathscr{D} .
$$

To confirm that $P$ satisfies either (33a) or (33b), it only remains to show that $F$ has the requisite continuity properties.

Now if $\lambda=0$, then $P(x)=F\left[P^{\phi}(x)\right]$ and, by the properties of $\pi, F$ is continuous on its entire domain, as required in (33a). Alternatively, suppose $\lambda \in(0,1)$ and define $V_{\lambda}:=\{\lambda+(1-\lambda) \phi(t)$ : $t \in D$ \}. Since $\phi$ is continuous and $\phi(z)=0$, it follows that $V_{\lambda}$ is an interval containing $\lambda$. Furthermore, if $V_{\lambda}=\{\lambda\}$ then $\phi(D)=\{0\}$ and $P^{\phi}$ is a trivial index, in which case (A21) yields

$$
P(x)=F[\lambda H(x)]<F\left[\lambda H\left(x^{\prime}\right)\right]=P\left(x^{\prime}\right) \text { whenever } H(x)<H\left(x^{\prime}\right),
$$

contradicting the assumption that (A9) is false. Hence $V_{\lambda}$ must be a nondegenerate interval. Furthermore, from (A2) we note that $F[u]$ is defined for all $u \in g[\mathscr{f}]$, where

$$
\begin{aligned}
g[\mathscr{P}] & :=\left\{g\left[P^{\phi}, H\right] \mid\left(P^{\phi}, H\right) \in \mathscr{S}\right\}=\left\{\lambda H+(1-\lambda) P^{\phi} \mid\left(P^{\phi}, H\right) \in \mathscr{P}\right\} \\
& =\{\lambda H+(1-\lambda) H \phi(t) \mid H \in \mathscr{H} ; t \in D\}=\left\{H \cdot v \mid H \in \mathscr{H} ; v \in V_{\lambda}\right\},
\end{aligned}
$$

and since $\pi\left[P^{\phi}, H\right]$ is continuous in $P^{\phi}$, we may deduce from (A21) that $F[H \cdot v]$ is continuous in $v$ on $V_{\lambda}$ for each $H \in \mathscr{H}$. It then follows that $F[u]$ is continuous for positive $u$, as required in (33b).

Finally we note that each of the cases (33a), (33b), and (33c) produces a subgroup consistent index satisfying (1)-(5). Hence Proposition 3 is established.

Proof of Corollary 4: Suppose $P$ is decomposable. Then setting $H_{J}=H_{k}=1$ and $\theta=\frac{1}{2}$ in (A8) yields

$$
f\left[\frac{1}{2} P_{J}+\frac{1}{2} P_{k}\right]=\frac{1}{2} f\left[P_{J}\right]+\frac{1}{2} f\left[P_{k}\right] \text { for all } P_{j}, P_{k} \in \phi(D),
$$

where $f\left[P^{\phi}\right]:=\pi\left[P^{\phi}, 1\right]$ is continuous and increasing in $P^{\phi}$. Equation (A24) is a Jensen equation whose solution (Aczel (1966, p. 46)) implies $f\left[P^{\phi}\right]=r P^{\phi}+s^{\prime}$ for some constants $r>0$ and $s^{\prime}$.

Now consider any $\left(P^{\phi}, H\right) \in \mathscr{\mathcal { S }}$, and let $P^{\phi}=\rho H$ for some $\rho \in \phi(D)$. If we choose $H_{J}=1$, $P_{j}=\rho, H_{k}=P_{k}=0$, and $\theta=H$ in (A8), and define $c:=\pi[0,0]$ and $s:=s^{\prime}-c$ we obtain

$$
\pi\left[P^{\phi}, H\right]=\pi[\rho, 1] H+\pi[0,0](1-H)=\left(r \rho+s^{\prime}\right) H+c(1-H)=r P^{\phi}+s H+c
$$

where $r>0$ and $s \geq 0$, since $\pi$ is nondecreasing in $H$. Hence there exist constants $c, r>0$ and $s \geq 0$ such that

$$
P(x)=r P^{\phi}(x)+s H(x)+c \text { for all } x \in \mathscr{D} .
$$

Finally, by setting $d(t)=r \phi(t)+s$ for $t \leq z$ and $d(t)=0$ for $t>z$, we find that $d$ satisfies (37), and that the corresponding canonical index satisfies $P^{d}=r P^{\phi}+s H$. Therefore $P=P^{d}+c$ for some canonical index $P^{d}$ and some constant $c$.

The converse follows trivially from the fact that any canonical index $P^{d}$ is decomposable, and hence the index $P^{d}+c$ is also decomposable. So Corollary 4 is established. 


\section{REFERENCES}

Aczel, J. (1966): Lectures on Functional Equations and Their Applications. New York: Academic Press.

Anand, S. (1983): Inequality and Poverty in Malaysia. Oxford: Oxford University Press.

Blackorby, C., and D. Donaldson (1980): "Ethical Indices for the Measurement of Poverty," Econometrica, 48, 1053-1060.

Blackorby, C., D. Primont and R. Russell (1978): Duality, Separability, and Functional Structure: Theory and Economic Applications. New York: North-Holland.

Chakravarty, S. R. (1983a): “A New Index of Poverty,” Mathematical Social Sciences, 6, 307-313. $74-85$.

Clark, S., R. Hemming, and D. Ulph (1981): "On Indices for the Measurement of Poverty," Economic Journal, 91, 515-526.

Donaldson, D., and J. A. Weymark (1986): "Properties of Fixed-Population Poverty Indices," International Economic Review, 27, 667-688.

Foster, J. E. (1984): “On Economic Poverty: A Survey of Aggregate Measures," in Advances in Econometrics, Vol. 3, ed. by R. L. Basmann and G. F. Rhodes. Connecticut: JAI Press.

Foster, J. E., J. Greer, AND E. Thorbecke (1984): “A Class of Decomposable Poverty Measures," Econometrica, 52, 761-766.

Gelbaum, B. R., And J. M. H. Olmsted (1964): Counterexamples in Analysis. San Francisco: Holden-Day.

Gorman, W. M. (1968): “The Structure of Utility Functions," Review of Economic Studies, 35, 367-390.

Kakwani, N. C. (1980): “On a Class of Poverty Measures." Econometrica, 48, 437-446.

MAK, K.-T. (1986): "On Separability: Functional Structure," Journal of Economic Theory, 40, $250-282$.

SEN, A. K. (1976): "Poverty: An Ordinal Approach to Measurement," Econometrica, 44, 219-231.

SHORROCKs, A. F. (1988): "Aggregation Issues in Inequality Measurement," in Measurement in Economics, ed. by W. Eichhorn. Heidelberg: Physica-Verlag.

TAKayama, N. (1979): "Poverty, Income Inequality and their Measures: Professor Sen's Axiomatic Approach Reconsidered," Econometrica, 47, 747-759.

Thon, D. (1979): “On Measuring Poverty,” Review of Income and Wealth, 25, 429-440. 
http://www.jstor.org

\title{
LINKED CITATIONS
}

- Page 1 of 4 -

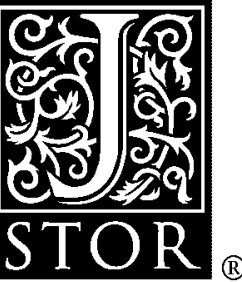

You have printed the following article:

Subgroup Consistent Poverty Indices

James E. Foster; Anthony F. Shorrocks

Econometrica, Vol. 59, No. 3. (May, 1991), pp. 687-709.

Stable URL:

http://links.jstor.org/sici?sici=0012-9682\%28199105\%2959\%3A3\%3C687\%3ASCPI\%3E2.0.CO\%3B2-Y

This article references the following linked citations. If you are trying to access articles from an off-campus location, you may be required to first logon via your library web site to access JSTOR. Please visit your library's website or contact a librarian to learn about options for remote access to JSTOR.

\section{[Footnotes]}

\author{
${ }^{2}$ A Class of Decomposable Poverty Measures \\ James Foster; Joel Greer; Erik Thorbecke \\ Econometrica, Vol. 52, No. 3. (May, 1984), pp. 761-766. \\ Stable URL: \\ http://links.jstor.org/sici?sici=0012-9682\%28198405\%2952\%3A3\%3C761\%3AACODPM\%3E2.0.CO\%3B2-O \\ ${ }^{3}$ Poverty: An Ordinal Approach to Measurement \\ Amartya Sen \\ Econometrica, Vol. 44, No. 2. (Mar., 1976), pp. 219-231. \\ Stable URL: \\ http://links.jstor.org/sici?sici=0012-9682\%28197603\%2944\%3A2\%3C219\%3APAOATM\%3E2.0.CO\%3B2-Z
}

\author{
${ }^{6}$ On a Class of Poverty Measures \\ Nanak Kakwani \\ Econometrica, Vol. 48, No. 2. (Mar., 1980), pp. 437-446. \\ Stable URL: \\ http://links.jstor.org/sici?sici=0012-9682\%28198003\%2948\%3A2\%3C437\%3AOACOPM\%3E2.0.CO\%3B2-H
}

\footnotetext{
${ }^{6}$ Poverty, Income Inequality, and Their Measures: Professor Sen's Axiomatic Approach Reconsidered

Noriyuki Takayama

Econometrica, Vol. 47, No. 3. (May, 1979), pp. 747-759.

Stable URL:

http://links.jstor.org/sici?sici=0012-9682\%28197905\%2947\%3A3\%3C747\%3APIIATM\%3E2.0.CO\%3B2-N

NOTE: The reference numbering from the original has been maintained in this citation list.
} 
http://www.jstor.org

\section{LINKED CITATIONS}

- Page 2 of 4 -

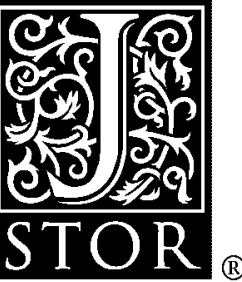

\section{${ }^{7}$ Properties of Fixed-Population Poverty Indices}

David Donaldson; John A. Weymark

International Economic Review, Vol. 27, No. 3. (Oct., 1986), pp. 667-688.

Stable URL:

http://links.jstor.org/sici?sici=0020-6598\%28198610\%2927\%3A3\%3C667\%3APOFPI\%3E2.0.CO\%3B2-R

\section{${ }^{8}$ Ethical Indices for the Measurement of Poverty}

Charles Blackorby; David Donaldson

Econometrica, Vol. 48, No. 4. (May, 1980), pp. 1053-1060.

Stable URL:

http://links.jstor.org/sici?sici=0012-9682\%28198005\%2948\%3A4\%3C1053\%3AEIFTMO\%3E2.0.CO\%3B2-M

\section{${ }^{8}$ Ethically Flexible Measures of Poverty}

Satya Ranjan Chakravarty

The Canadian Journal of Economics / Revue canadienne d'Economique, Vol. 16, No. 1. (Feb., 1983), pp. 74-85.

Stable URL:

http://links.jstor.org/sici?sici=0008-4085\%28198302\%2916\%3A1\%3C74\%3AEFMOP\%3E2.0.CO\%3B2-U

\section{${ }^{8}$ On Indices for the Measurement of Poverty}

Stephen Clark; Richard Hemming; David Ulph

The Economic Journal, Vol. 91, No. 362. (Jun., 1981), pp. 515-526.

Stable URL:

http://links.jstor.org/sici?sici=0013-0133\%28198106\%2991\%3A362\%3C515\%3AOIFTMO\%3E2.0.CO\%3B2-I

\section{References}

\section{Ethical Indices for the Measurement of Poverty}

Charles Blackorby; David Donaldson

Econometrica, Vol. 48, No. 4. (May, 1980), pp. 1053-1060.

Stable URL:

http://links.jstor.org/sici?sici=0012-9682\%28198005\%2948\%3A4\%3C1053\%3AEIFTMO\%3E2.0.CO\%3B2-M

NOTE: The reference numbering from the original has been maintained in this citation list. 
http://www.jstor.org

\section{LINKED CITATIONS}

- Page 3 of 4 -

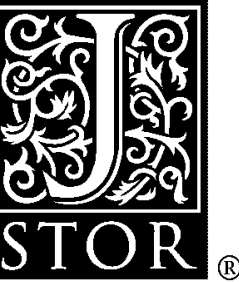

\section{Ethically Flexible Measures of Poverty}

Satya Ranjan Chakravarty

The Canadian Journal of Economics / Revue canadienne d'Economique, Vol. 16, No. 1. (Feb., 1983), pp. 74-85.

Stable URL:

http://links.jstor.org/sici?sici=0008-4085\%28198302\%2916\%3A1\%3C74\%3AEFMOP\%3E2.0.CO\%3B2-U

\section{On Indices for the Measurement of Poverty}

Stephen Clark; Richard Hemming; David Ulph

The Economic Journal, Vol. 91, No. 362. (Jun., 1981), pp. 515-526.

Stable URL:

http://links.jstor.org/sici?sici=0013-0133\%28198106\%2991\%3A362\%3C515\%3AOIFTMO\%3E2.0.CO\%3B2-I

\section{Properties of Fixed-Population Poverty Indices}

David Donaldson; John A. Weymark

International Economic Review, Vol. 27, No. 3. (Oct., 1986), pp. 667-688.

Stable URL:

http://links.jstor.org/sici?sici=0020-6598\%28198610\%2927\%3A3\%3C667\%3APOFPI\%3E2.0.CO\%3B2-R

\section{A Class of Decomposable Poverty Measures}

James Foster; Joel Greer; Erik Thorbecke

Econometrica, Vol. 52, No. 3. (May, 1984), pp. 761-766.

Stable URL:

http://links.jstor.org/sici?sici=0012-9682\%28198405\%2952\%3A3\%3C761\%3AACODPM\%3E2.0.CO\%3B2-O

\section{The Structure of Utility Functions}

W. M. Gorman

The Review of Economic Studies, Vol. 35, No. 4. (Oct., 1968), pp. 367-390.

Stable URL:

http://links.jstor.org/sici?sici=0034-6527\%28196810\%2935\%3A4\%3C367\%3ATSOUF\%3E2.0.CO\%3B2-6

\section{On a Class of Poverty Measures}

Nanak Kakwani

Econometrica, Vol. 48, No. 2. (Mar., 1980), pp. 437-446.

Stable URL:

http://links.jstor.org/sici?sici=0012-9682\%28198003\%2948\%3A2\%3C437\%3AOACOPM\%3E2.0.CO\%3B2-H

NOTE: The reference numbering from the original has been maintained in this citation list. 
http://www.jstor.org

\section{LINKED CITATIONS \\ - Page 4 of 4 -}

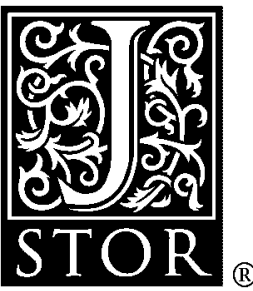

\section{Poverty: An Ordinal Approach to Measurement}

Amartya Sen

Econometrica, Vol. 44, No. 2. (Mar., 1976), pp. 219-231.

Stable URL:

http://links.jstor.org/sici?sici=0012-9682\%28197603\%2944\%3A2\%3C219\%3APAOATM\%3E2.0.CO\%3B2-Z

Poverty, Income Inequality, and Their Measures: Professor Sen's Axiomatic Approach Reconsidered

Noriyuki Takayama

Econometrica, Vol. 47, No. 3. (May, 1979), pp. 747-759.

Stable URL:

http://links.jstor.org/sici?sici=0012-9682\%28197905\%2947\%3A3\%3C747\%3APIIATM\%3E2.0.CO\%3B2-N 